\title{
Development and validation of Nomograms for predicting overall survival and Cancer-specific survival in patients with ovarian clear cell carcinoma
}

Qian Chen, Shu Wang ${ }^{*}$ (D) and Jing-He Lang ${ }^{*}$

\begin{abstract}
Background: Ovarian clear cell carcinoma (OCCC) is a rare histologic type of ovarian cancer. There is a lack of an efficient prognostic predictive tool for OCCC in clinical work. This study aimed to construct and validate nomograms for predicting the overall survival (OS) and cancer-specific survival (CSS) in patients with OCCC.

Methods: Data of patients with primary diagnosed OCCC in the Surveillance, Epidemiology, and End Results (SEER) database between 2010 and 2016 was extracted. Prognostic factors were evaluated with LASSO Cox regression and multivariate Cox regression analysis, which were applied to construct nomograms. The performance of the nomogram models was assessed by the concordance index (C-index), calibration plots, decision curve analysis (DCA) and risk subgroup classification. The Kaplan-Meier curves were plotted to compare survival outcomes between subgroups.

Results: A total of 1541 patients from SEER registries were randomly divided into a training cohort $(n=1079)$ and a validation cohort $(n=462)$. Age, laterality, stage, lymph node $(\mathrm{LN})$ dissected, organ metastasis and chemotherapy were independently and significantly associated with OS, while laterality, stage, LN dissected, organ metastasis and chemotherapy were independent risk factors for CSS. Nomograms were developed for the prediction of 3- and 5-year OS and CSS. The C-indexes for OS and CSS were 0.802[95\% confidence interval (Cl) $0.773-0.831$ ] and 0.802 (0.769$0.835)$, respectively, in the training cohort, while $0.746(0.691-0.801)$ and $0.770(0.721-0.819)$, respectively, in the validation cohort. Calibration plots illustrated favorable consistency between the nomogram predicted and actual survival. C-index and DCA curves also indicated better performance of nomogram than the AJCC staging system. Significant differences were observed in the survival curves of different risk subgroups.

Conclusions: We have constructed predictive nomograms and a risk classification system to evaluate the OS and CSS of OCCC patients. They were validated to be of satisfactory predictive value, and could aid in future clinical practice.
\end{abstract}

Keywords: Ovarian clear cell carcinoma, Nomogram, Predict, Overall survival, Cancer-specific survival

*Correspondence: wangshu219@hotmail.com; langjh@hotmail.com Department of Obstetrics and Gynecology, Peking Union Medical College Hospital, Chinese Academy of Medical Science and Peking Union Medical College, 1 ShuaiFuYuan, Wangfujing, DongCheng District, Beijing 100730, P.R. China

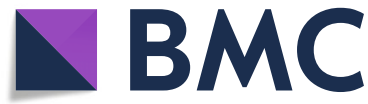

\section{Introduction}

Ovarian cancer $(\mathrm{OC})$ is one of the most aggressive gynecological cancer, consisting of a group of heterogonous tumors. As a subtype of epithelial ovarian cancer (EOC), ovarian clear cell carcinoma (OCCC) presents a distinct biological profile from other histological types [1]. With a higher

(c) The Author(s). 2020 Open Access This article is licensed under a Creative Commons Attribution 4.0 International License, which permits use, sharing, adaptation, distribution and reproduction in any medium or format, as long as you give appropriate credit to the original author(s) and the source, provide a link to the Creative Commons licence, and indicate if changes were made. The images or other third party material in this article are included in the article's Creative Commons licence, unless indicated otherwise in a credit line to the material. If material is not included in the article's Creative Commons licence and your intended use is not permitted by statutory regulation or exceeds the permitted use, you will need to obtain permission directly from the copyright holder. To view a copy of this licence, visit http://creativecommons.org/licenses/by/4.0/. The Creative Commons Public Domain Dedication waiver (http://creativecommons.org/publicdomain/zero/1.0/) applies to the data made available in this article, unless otherwise stated in a credit line to the data. 
incidence in East Asia ( 30\%), OCCC is reported to be diagnosed at a younger age compared with serous carcinoma [2].

Patients with early-stage OCCC generally exhibit favorable prognosis, while those in advanced stage present worse survival outcomes than patients in the high-grade serous group [3]. In addition to stage, other factors were proposed to exert influences on the prognosis of OCCC, such as the presence of endometriosis, surgical methods and venous thromboembolism $[4,5]$. Demographic characteristics, tumor size, lymph node status and treatment strategies are also important when evaluating survival outcomes.

Standard treatment guidelines for OCCC have not yet been developed, given its rarity in the large clinical trials of EOC. A comprehensive prognostic judgment system would be useful to guide the selection of a treatment protocol. The nomogram, a statistic-based predictive tool with the ability to integrate pivotal predictive factors, has been widely utilized to quantify risks and evaluate the prognosis of many cancer types [6-8]. However, to the best of our knowledge, no nomograms for patients with OCCC have been developed. In this study, we aimed to construct nomograms using data extracted from the Surveillance, Epidemiology, and End Results (SEER) database to predict the prognosis of patients with OCCC.

\section{Materials and methods \\ Data source}

Medical records of patients with OCCC were obtained from the SEER database, which contains data of cancer patients from 18 regional registries, covering approximately 34.6\% of the total population in the United States [9]. Relevant information was extracted applying SEER*Stat software version 8.3.6 (https://seer.cancer.gov/seerstat/).

\section{Data extraction}

Patients diagnosed as OCCC from 2010 to 2016 were selected through the International Classification of Diseases for Oncology, 3rd edition (ICD-O-3) morphology codes "8310/3; 8313/3; 8443/3; 8444/3" from OC patients. Variables for this study included age at diagnosis, race, laterality, grade, stage (American Joint Commission on Cancer [AJCC] 7th version), tumor size, organ metastasis, radiotherapy, chemotherapy, number of examined lymph nodes (LNs), LN status, vital status and survival time. The organ metastasis sites referred to liver, lung, bone, and brain according to the available data in the SEER database.

Patients with OCCC were excluded in the following scenarios: (1) not the primary tumor; (2) without histologic confirmation; (3) survival time shorter than 1 month; (4) no surgery; and (5) unknown information about LN, race, tumor size, stage and organ metastasis.

\section{Statistic methods}

Patients enrolled in our study were randomly assigned into the training cohort and validation cohort at a ratio of 7:3. The primary end points were overall survival (OS) and cancer-specific survival (CSS). Categorical variables were shown as frequencies and proportions. The comparison of clinicopathological characteristics between the training and validation cohorts was performed using a chi-squared test.

The least absolute shrinkage and selection operator (LASSO) method was used to primarily select useful predictive features to avoid over-fitting to some extent. Significant prognostic factors were further identified in multivariate analysis from the Cox proportional hazards model. Then, the nomograms associated with OS and CSS were constructed incorporating the final risk factors.

The performance of the nomogram was validated internally in the training cohort and externally in the validation cohort. Harrell's concordance index (C-index) ranging from 0.5 to 1.0 was used to evaluate the discriminative abilities of the nomograms. Calibration curves (1000 bootstrap resamples) were generated to test the consistency between the predicted and actual 3- and 5year OS and CSS. Emerging as a new method, decision curve analysis (DCA) was applied to evaluate the latent value of the nomograms [10]. Moreover, the whole cohort was regrouped into low- and high-risk groups with the median risk score generated from the nomogram. Kaplan-Meier analysis and log-rank test were used to explore the survival difference between risk subgroups.

All statistical analyses were performed with SPSS (version 25.0, SPSS, Chicago, IL, USA) and R software (version 3.6.0; http://www.r-project.org/). A $P$ value of $<0.05$ was considered statistically significant.

\section{Results}

\section{Patient characteristics and survival outcomes}

A total of 1541 patients diagnosed with primary OCCC were identified from the SEER database. Most of the patients were at an early stage $(78.3 \%)$ and the proportion of white patients (74.9\%) was the greatest. 1223(79.4\%) patients underwent lymph node dissection and 1265 (82.1\%) received chemotherapy. Characteristics of patients in the training cohort $(n=1079)$ and the validation cohort $(n=462)$ were listed in Table 1 .

The 3- and 5-year OS rates were 77.8 , and $70.6 \%$ for all patients, respectively, with a mean follow-up time of 65.3 months. The 3- and 5-year CSS rates were 77.8, and $70.6 \%$ for all patients, respectively, with a mean followup time of 66.8 months. The 3- and 5-year OS and CSS rates of all patients in terms of different clinical features were shown in Table 2. 
Table 1 Patients' demographics and clinicopathological characteristics

\begin{tabular}{|c|c|c|c|c|}
\hline Variables & All patients $\mathrm{n}(\%)$ & Training set $\mathbf{n}(\%)$ & Validation set $\mathbf{n}(\%)$ & $P$ \\
\hline Total & $1541(100.0)$ & $1079(70.0)$ & $462(30.0)$ & \\
\hline Age, years & & & & 0.984 \\
\hline$<50$ & $413(26.8)$ & $290(26.9)$ & $124(26.8)$ & \\
\hline $50-60$ & $609(39.5)$ & $425(39.4)$ & $184(39.8)$ & \\
\hline$>60$ & $518(33.6)$ & $364(33.7)$ & $154(33.3)$ & \\
\hline Tumor size, cm & & & & 0.325 \\
\hline$\leq 12$ & $810(52.6)$ & $576(53.4)$ & $234(50.6)$ & \\
\hline$>12$ & $731(47.4)$ & $503(46.6)$ & $228(49.4)$ & \\
\hline Race & & & & 0.524 \\
\hline White & $1154(74.9)$ & $813(75.3)$ & $341(73.8)$ & \\
\hline Other & $387(25.1)$ & $266(24.7)$ & $121(26.2)$ & \\
\hline Laterality & & & & 0.289 \\
\hline Unilateral & $1374(89.2)$ & $968(89.7)$ & $406(87.9)$ & \\
\hline Bilateral & $167(10.8)$ & $111(10.3)$ & $56(12.1)$ & \\
\hline AJCC Stage (7th) & & & & 0.730 \\
\hline । & $979(67.5)$ & $687(63.7)$ & $292(63.2)$ & \\
\hline$\|$ & $167(10.8)$ & $111(10.3)$ & $56(12.1)$ & \\
\hline III & $302(19.6)$ & $214(19.8)$ & $88(19.0)$ & \\
\hline IV & $395(6.0)$ & $67(6.2)$ & $26(5.6)$ & \\
\hline Grade & & & & 0.167 \\
\hline$|/| \mid$ & $121(7.9)$ & $87(8.1)$ & $34(7.4)$ & \\
\hline III & $588(38.2)$ & $429(39.8)$ & $159(34.4)$ & \\
\hline IV & $398(25.8)$ & $266(24.7)$ & $132(28.6)$ & \\
\hline Unknown & $434(28.2)$ & $297(27.5)$ & $137(29.7)$ & \\
\hline LN dissected, $\mathrm{n}$ & & & & 0.154 \\
\hline No & $318(20.6)$ & $217(20.1)$ & $101(21.9)$ & \\
\hline $1-10$ & $446(28.9)$ & $328(30.4)$ & $118(25.5)$ & \\
\hline$>10$ & $777(50.4)$ & $534(49.5)$ & $243(52.6)$ & \\
\hline LN & & & & 0.233 \\
\hline No dissection or Negative & $1370(88.9)$ & $966(89.5)$ & $404(87.4)$ & \\
\hline Positive & $171(11.1)$ & $113(10.5)$ & $58(12.6)$ & \\
\hline Organ Metastasis & & & & 0.939 \\
\hline No & $1505(97.7)$ & $1054(97.7)$ & $451(97.6)$ & \\
\hline Yes & $36(2.3)$ & $25(2.3)$ & $11(2.4)$ & \\
\hline Radiotherapy & & & & 0.312 \\
\hline No/Unknown & 1509 (97.9) & $1054(97.7)$ & $451(98.5)$ & \\
\hline Yes & $32(2.1)$ & $25(2.3)$ & $7(1.5)$ & \\
\hline Chemotherapy & & & & 0.800 \\
\hline No/Unknown & $276(17.9)$ & $195(18.1)$ & $81(17.5)$ & \\
\hline Yes & $1265(82.1)$ & $884(81.9)$ & $381(82.5)$ & \\
\hline
\end{tabular}

AJCC American Joint Committee on Cancer, $L N$ lymph node 
Table 2 The 3-and 5-year overall survival and cancer-specific survival in terms of patient characteristics

\begin{tabular}{|c|c|c|c|c|}
\hline \multirow[t]{2}{*}{ Variables } & \multicolumn{2}{|c|}{ Overall Survival } & \multicolumn{2}{|c|}{ Cancer-Specific Survival } \\
\hline & 3-year (\%) & 5-year (\%) & 3-year (\%) & 5-year (\%) \\
\hline \multicolumn{5}{|l|}{ Age, years } \\
\hline$<50$ & 73.4 & 66.3 & 74.4 & 67.8 \\
\hline $50-60$ & 82.0 & 73.7 & 82.9 & 75.3 \\
\hline$>60$ & 76.4 & 70.2 & 78.6 & 73.4 \\
\hline \multicolumn{5}{|l|}{ Tumor size, $\mathrm{cm}$} \\
\hline$\leq 12$ & 77.4 & 72.8 & 79.0 & 75.0 \\
\hline$>12$ & 78.4 & 68.4 & 79.6 & 70.4 \\
\hline \multicolumn{5}{|l|}{ Race } \\
\hline White & 79.2 & 72.5 & 80.7 & 74.8 \\
\hline Black & 57.7 & 40.3 & 61.3 & 42.8 \\
\hline Other & 76.8 & 68.8 & 77.4 & 70.0 \\
\hline \multicolumn{5}{|l|}{ Laterality } \\
\hline Unilateral & 81.8 & 75.1 & 83.1 & 77.2 \\
\hline Bilateral & 46.4 & 35.5 & 48.4 & 37.1 \\
\hline \multicolumn{5}{|l|}{ AJCC Stage (7th) } \\
\hline । & 91.2 & 85.6 & 92.2 & 87.2 \\
\hline$\|$ & 76.9 & 74.5 & 78.3 & - \\
\hline III & 46.7 & 34.1 & 49.0 & 35.8 \\
\hline IV & 40.8 & 27.9 & 41.7 & 30.5 \\
\hline \multicolumn{5}{|l|}{ Grade } \\
\hline$|/| \mid$ & 85.4 & 73.6 & 86.3 & 79.2 \\
\hline III & 75.9 & 67.4 & 77.0 & 69.2 \\
\hline IV & 78.5 & 75.0 & 80.9 & 77.3 \\
\hline Unknown & - & - & - & - \\
\hline \multicolumn{5}{|l|}{ LN dissected, $\mathrm{n}$} \\
\hline No & 64.1 & 59.7 & 66.7 & 63.3 \\
\hline $1-10$ & 78.4 & 70.6 & 79.6 & 72.7 \\
\hline$>10$ & 83.1 & 75.1 & 84.2 & 76.6 \\
\hline \multicolumn{5}{|l|}{ LN } \\
\hline No dissection or Negative & 78.5 & 70.7 & 79.9 & 72.6 \\
\hline Positive & 72.5 & 69.8 & 74.3 & 70.1 \\
\hline \multicolumn{5}{|l|}{ Organ Metastasis } \\
\hline No & 79.0 & 71.8 & 80.5 & 73.9 \\
\hline Yes & 30.5 & 22.2 & 30.5 & 26.7 \\
\hline \multicolumn{5}{|l|}{ Radiotherapy } \\
\hline No/Unknown & 78.0 & 70.7 & 79.5 & 72.9 \\
\hline Yes & 66.4 & 33.2 & 66.4 & - \\
\hline \multicolumn{5}{|l|}{ Chemotherapy } \\
\hline No/Unknown & 80.5 & 76.1 & 83.4 & 80.5 \\
\hline Yes & 77.2 & 69.4 & 78.4 & 71.1 \\
\hline
\end{tabular}




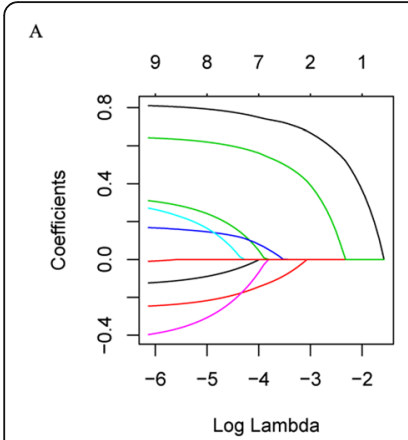

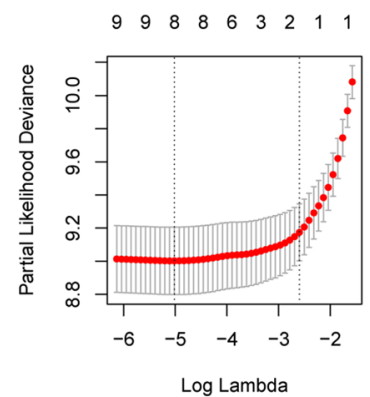

C

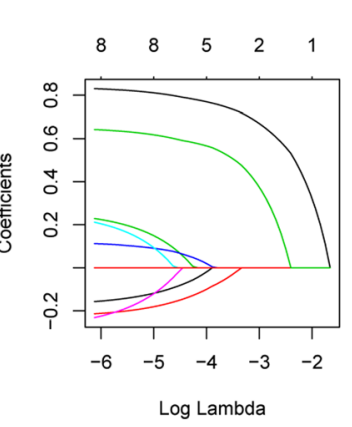

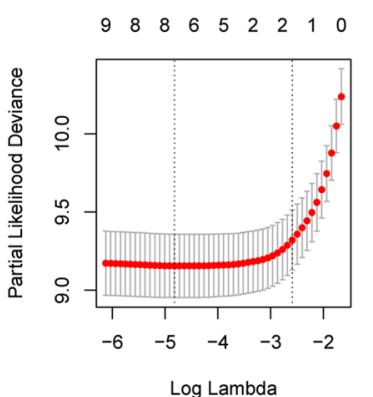

Fig. 1 The LASSO regression used to select prognostic factors for OS and CSS; a LASSO coefficient profiles of 11 variables for OS; b LASSO Cox analysis identified 8 variables for OS; c LASSO coefficient profiles of 11 variables for CSS; $\mathbf{d}$ LASSO Cox analysis identified 8 variables for CSS. OS: overall survival; CSS: cancer-specific survival

Table 3 Multivariate Cox analysis of the training cohort based on the results of lasso regression

\begin{tabular}{|c|c|c|c|c|}
\hline & \multicolumn{2}{|l|}{ OS } & \multicolumn{2}{|l|}{ CSS } \\
\hline & HR $(95 \% \mathrm{Cl})$ & $\mathbf{P}$ & $\mathrm{HR}(95 \% \mathrm{Cl})$ & $\mathbf{P}$ \\
\hline \multicolumn{5}{|l|}{ Age, years } \\
\hline$<50$ & Reference & & Reference & \\
\hline $50-60$ & $0.694(0.494-0.974)$ & 0.035 & $0.714(0.501-1.018)$ & 0.063 \\
\hline$>60$ & $0.781(0.556-1.098)$ & 0.155 & $0.735(0.511-1.057)$ & 0.097 \\
\hline \multicolumn{5}{|l|}{ Laterality } \\
\hline Unilateral & Reference & & Reference & \\
\hline Bilateral & 1.789 (1.283-2.496) & $<0.001$ & $1.792(1.265-2.538)$ & 0.001 \\
\hline \multicolumn{5}{|l|}{ AJCC Stage (7th) } \\
\hline । & Reference & & Reference & \\
\hline$\|$ & 3.039 (1.806-5.116) & $<0.001$ & $3.035(1.725-5.341)$ & $<0.001$ \\
\hline III & $8.683(6.015-12.536)$ & $<0.001$ & $9.168(6.204-13.549)$ & $<0.001$ \\
\hline IV & $7.466(4.318-12.909)$ & $<0.001$ & $7.862(4.392-14.073)$ & $<0.001$ \\
\hline \multicolumn{5}{|l|}{ LN dissected, $\mathrm{n}$} \\
\hline No & Reference & & Reference & \\
\hline $1-10$ & $0.738(0.517-1.054)$ & 0.094 & $0.765(0.524-1.118)$ & 0.167 \\
\hline$>10$ & $0.565(0.403-0.794)$ & $<0.001$ & $0.597(0.416-0.856)$ & 0.005 \\
\hline \multicolumn{5}{|l|}{ LN } \\
\hline No dissection or Negative & Reference & & Reference & \\
\hline Positive & $1.341(0.884-2.035)$ & 0.168 & $1.247(0.794-1.958)$ & 0.337 \\
\hline \multicolumn{5}{|l|}{ Organ Metastasis } \\
\hline No & Reference & & Reference & \\
\hline Yes & $2.326(1.203-4.497)$ & 0.012 & $2.256(1.123-4.535)$ & 0.022 \\
\hline \multicolumn{5}{|l|}{ Radiotherapy } \\
\hline No/Unknown & Reference & & Reference & \\
\hline Yes & $1.598(0.776-3.287)$ & 0.203 & $1.526(0.705-3.301)$ & 0.283 \\
\hline \multicolumn{5}{|l|}{ Chemotherapy } \\
\hline No/Unknown & Reference & & Reference & \\
\hline Yes & $0.531(0.362-0.780)$ & 0.001 & $0.615(0.401-0.944)$ & 0.026 \\
\hline
\end{tabular}



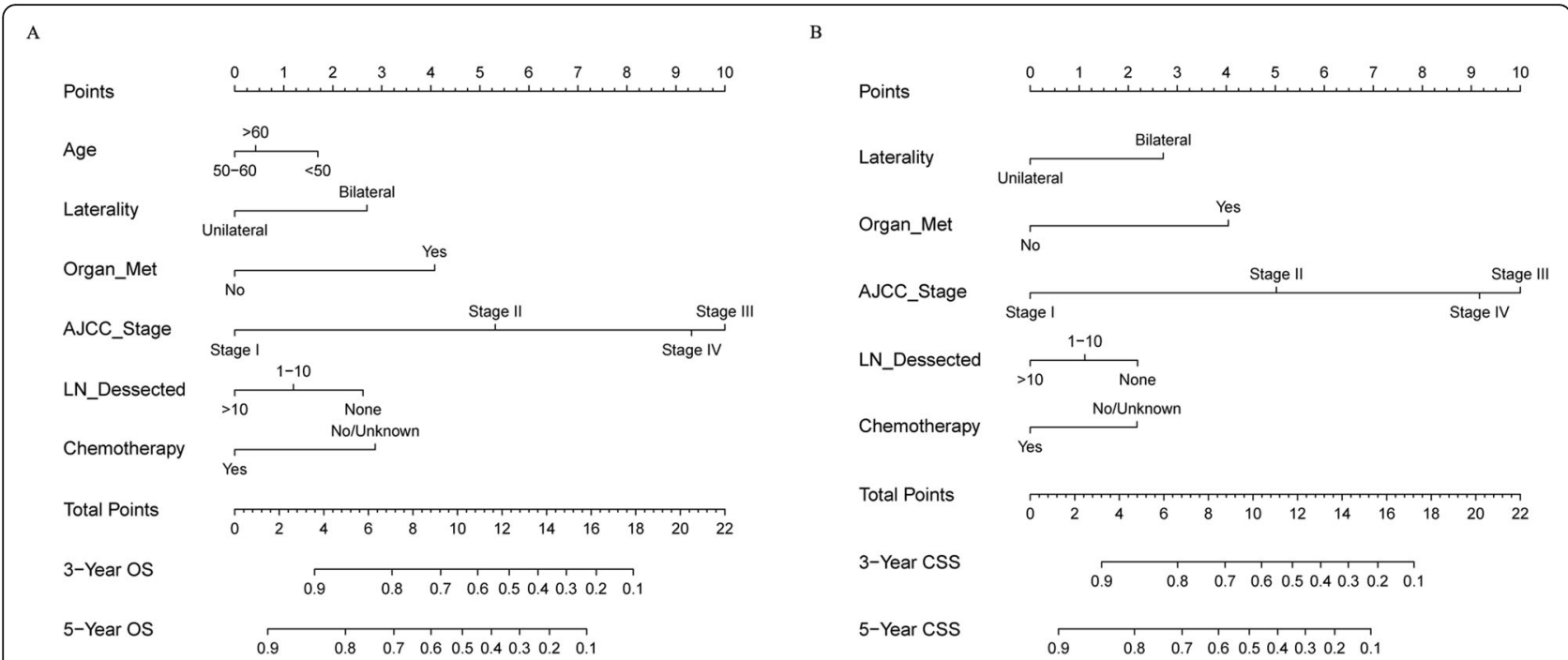

Fig. 2 Predictive nomograms. a Nomogram for predicting 3- and 5-year OS; b Nomogram for predicting 3- and 5-year CSS. Met: metastasis; AJCC: American Joint Commission on Cancer; LN: Iymph node; OS: overall survival; CSS: cancer-specific survival

\section{Construction of the prognostic nomograms for OS and CSS}

In total, 11 variables were included in the analysis. According to the results of LASSO Cox regression analysis, age, laterality, stage, LN dissected, LN status, organ metastasis, radiotherapy and chemotherapy were identified for OS and CSS risk factors (Fig. 1). In the multivariate analysis of these 8 factors, age, laterality, stage, LN dissected, organ metastasis and chemotherapy were independently and significantly associated with OS, while laterality, stage, LN dissected, organ metastasis and chemotherapy were independently and significantly associated with CSS (Table 3). Based on the above, nomograms were constructed by incorporating the prognostic factors to predict 3- and 5-year OS and CSS (Fig. 2).

\section{Nomogram validation}

The C-indexes for the nomogram of OS and CSS in the training cohort were $0.802[95 \%$ confidence interval (CI) 0.773-0.831] and $0.802(0.769-0.835)$, respectively, both of which were greater than the AJCC staging system [OS:0.775 (0.746-0.804); CSS: $0.781(0.750-0.813)]$. In the validation cohort, the C-indexes for the new model of OS and CSS [0.746 (0.691-0.801) and $0.770(0.721-0.819)$, respectively] also presented superiority over the AJCC staging system [OS: 0.731 (0.684-0.778); CSS: 0.752 (0.707-0.791)].

The calibration curves indicated excellent agreement between the nomogram predicted and actual survival outcomes in the training and validation cohort (Fig. 3). DCA curves indicated that the nomogram models made favorable predictions and outperformed the AJCC staging system (Fig. 4).
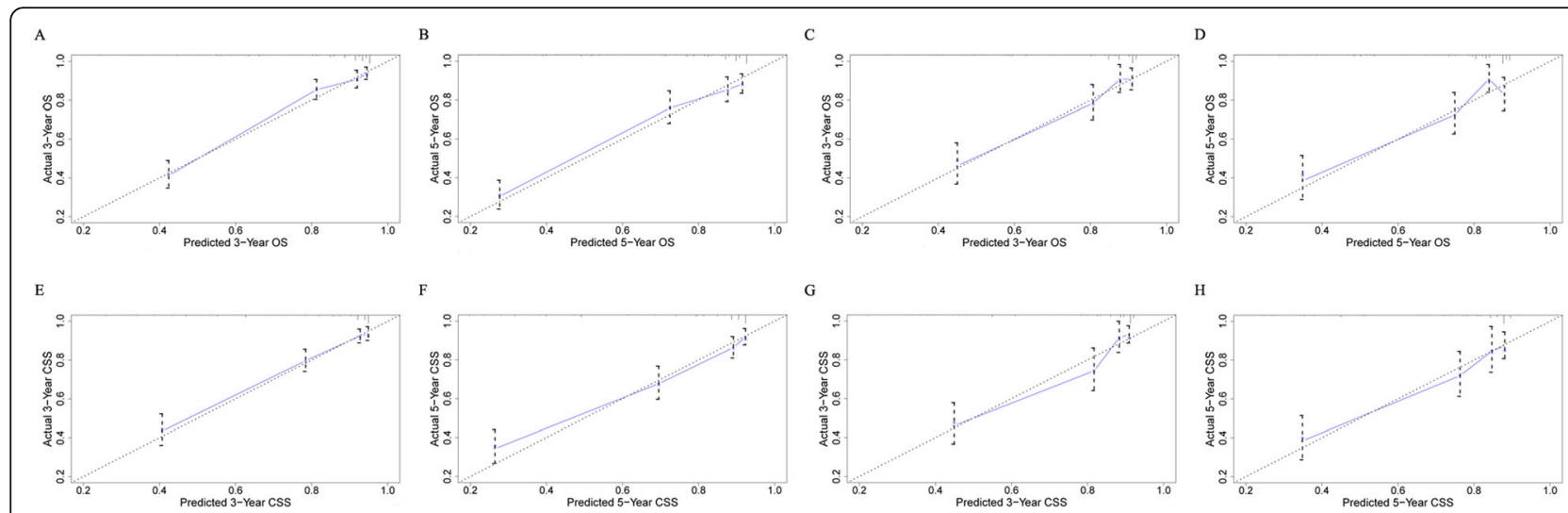

G

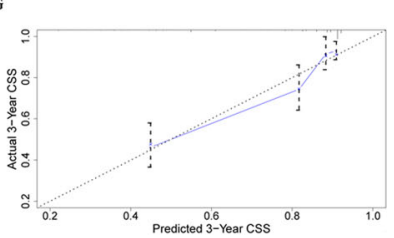

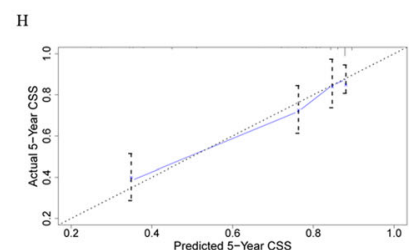

Fig. 3 Calibration plots. a 3-year and (b) 5-year OS nomogram calibration plots for training cohort; c 3-year and (d) 5-year OS nomogram calibration plots for validation cohort; e 3-year and (f) 5-year CSS nomogram calibration plots for training cohort; $\mathbf{g}$ 3-year and (H) 5-year CSS nomogram calibration plots for validation cohort. OS: overall survival; CSS: cancer-specific survival 

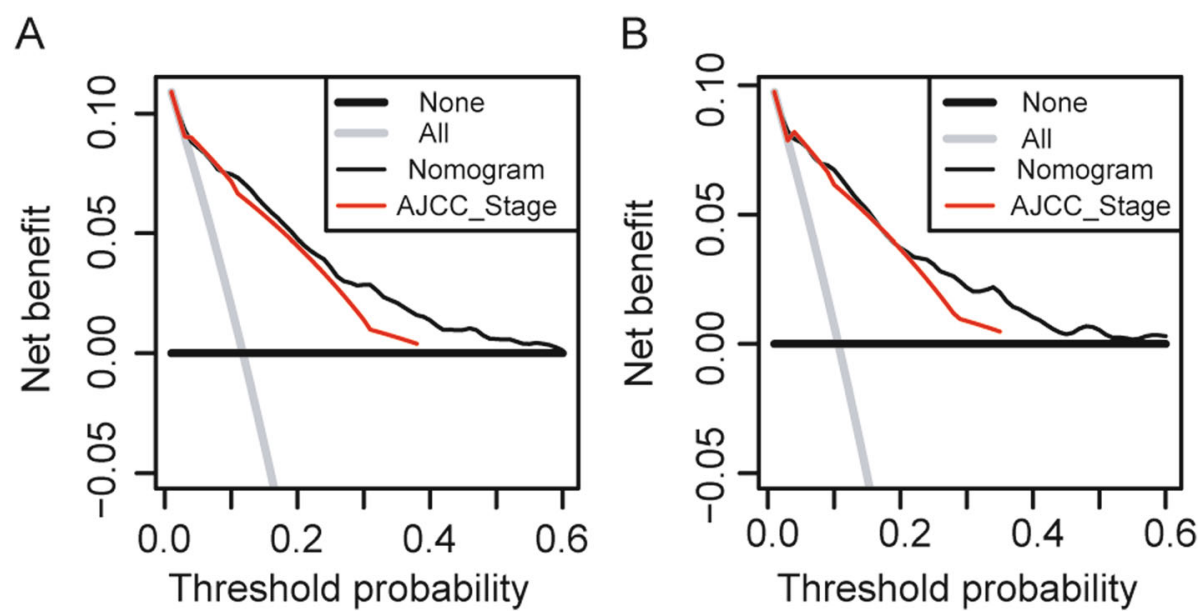

Fig. 4 DCA curve of the nomogram and AJCC stage for (a) OS and (b) CSS. DCA: decision curve analysis; AJCC: American Joint Commission on Cancer; OS: overall survival; CSS: cancer-specific survival

\section{Risk stratification of OCCC patients}

The risk score of each variable was generated from the nomogram and the total scores were calculated for all the patients. The median risk score was 6 (range: 3-21) for OS and 4 (range: 2-19) for CSS. The whole cohort was divided into low- and high- risk subgroups based on the median risk score. According to the survival curves in Fig. 5, significant differences were observed between the low- and high -risk groups for both OS $(P<0.001)$ and CSS $(P<0.001)$, implying the nomogram's outstanding ability for risk stratification.

\section{Discussion}

In the current study, data of patients with OCCC who have undergone surgery in the SEER database were used for the analysis of risk factors. Nomograms were constructed to assess the 3- and 5-year CSS and OS based on the identified prognostic factors. Favorable discrimination and calibration were observed from $\mathrm{C}$-index, calibration curves and DCA curves in both training and validation sets, indicating excellent performances of the nomograms. Moreover, risk scores generated from the nomograms were applied to successfully build a risk stratification system.

Our study identified six independent prognostic factors for OS: age, tumor laterality, organ metastasis, LN dissected, stage and chemotherapy. These factors also significantly impact CSS, except for age. Generally, patients at an older age are more likely to present worse survival outcomes due to lower immune response [11]. However, we observed that patients younger than 50 years tended to have poorer prognosis. One of the possible explanations may be the relatively conservative surgical mode for patients who wanted to preserve fertility. In addition to age, the relationship between other demographic characteristics (such as race) and prognosis was also explored.

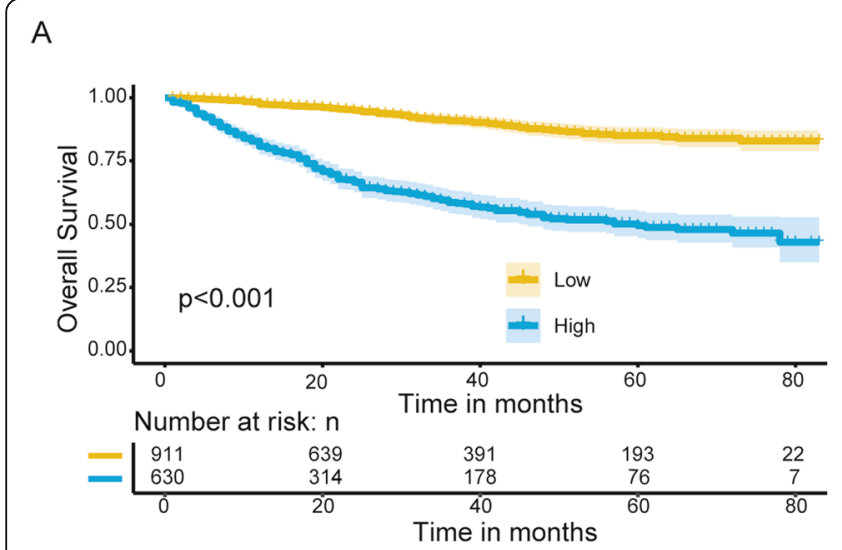

B

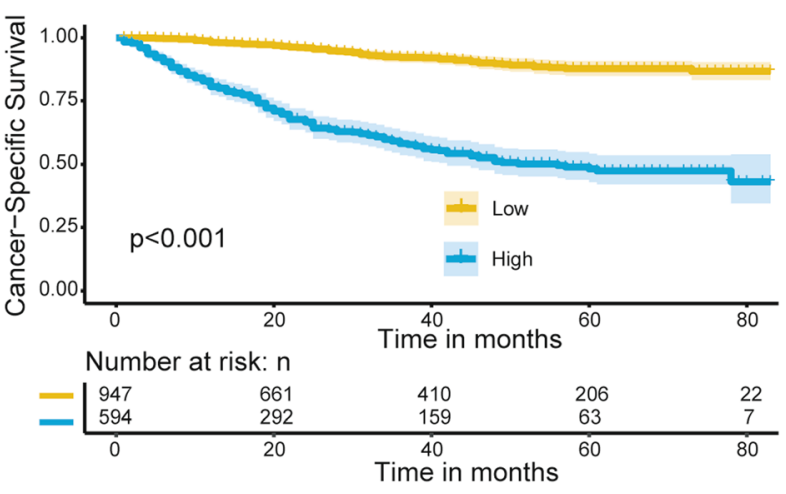

Fig. 5 Kaplan-Meier curves. a Overall survival and (b) cancer-specific survival for patients stratified by the risk stratification system 
Systematic lymphadenectomy was regarded as an important part of treatment guidelines for patients with EOC considering the prognostic value of LN status [12]. We observed that patients with more than 10 lymph nodes removed were associated with better prognosis. A 10-lymph node cutoff was defined as adequate lymphadenectomy according to the Gynecologic Oncology Group criteria. Several retrospective studies have demonstrated favorable survival outcomes of systematic lymphadenectomy on patients with early-stage OCCC [13-15]. In a combined exploratory analysis of three prospectively randomized phase III multicenter trials, Magazzino et al. reported that lymphadenectomy offered benefit to patients with advanced $\mathrm{OC}$ who received complete intraperitoneal debulking [16]. However, another study did not observe significant improvement in the survival of advanced OCCC patients with systematic retroperitoneal lymphadenectomy [17]. It is worth noting that LN status was not a prognostic factor in our study. Therefore, the role of lymphadenectomy on the whole cohort of OCCC patients required further investigation.

Chemotherapy is important in the management of EOC, especially for high-grade cases, while the role of chemotherapy for patients with OCCC remains controversial [18]. It was reported that the response rate of OCCC to conventional platinum was much lower than serous type in the first-line setting [19]. Published studies mainly focused on the performance of chemotherapy on early-stage cases [20-22]. In our study, we noted that chemotherapy was significantly associated with OS and CSS, implying its value in improving survival outcomes. Toru and his group carried out a randomized phase III trial (JGOG3017/GCIG Trial) to make a comparison between two chemotherapy regimens for OCCC. They demonstrated that irinotecan plus cisplatin and paclitaxel plus carboplatin were both well tolerated with no significant difference in survival benefit [23].

Several studies have reported better performance of the nomogram model than conventional staging systems and proposed it as a promising tool for prognosis evaluation [24-26]. Diao and his group developed a nomogram to predict OS in patients with inflammatory breast cancer, with a C-index of $0.738(0.717-0.759,24]$. Zhou et al. generated nomograms to predict the CSS and OS for stage IIII colon cancer patients, achieving a C-index of 0.78 (0.77-0.80) for CSS and 0.74 (0.73-0.75) for OS [25]. Similarly, a prediction model was constructed for patients with non-small cell lung cancer, with a C-index of 0.674 $(0.652-0.696,26]$. All of the above nomograms presented better discriminatory capacity than did the staging systems. Lack of some clinical information was the common limitation for these studies.

The nomograms developed in our study also presented better prediction capacity than AJCC 7 th staging system.
The nomogram model enables risk stratification of patients, thus facilitating personalized treatment plans and follow-up schedules. Considering the chemo-resistant feature of OCCC, efforts have been made to explore precision medicine based on molecular profiles, such as drugs targeting ARID1A-deficient OCCC patients [27]. It may be feasible to use a prediction model to select candidates for clinical trials.

It should be noted that there are several limitations in our study. First, detailed information about chemotherapy and radiotherapy as well as surgical procedures were unavailable. Data about the recurrence and reoperation were also unavailable in the SEER database. Second, selection bias was inevitable due to the study's retrospective nature. Third, the nomogram model only received internal validation. External validation of cohorts from other countries and prospective randomized clinical trials are were necessary to confirm its performance.

\section{Conclusion}

Nomograms with favorable capacity of prognosis assessment of 3- and 5-year OS and CSS for patients with initially diagnosed OCCC were constructed using data from a large-scale dataset. A risk stratification system was built based on risk scores generated from the nomograms. These nomograms may be useful to provide prognostic information in clinical work.

\section{Acknowledgements}

None.

Authors' contributions

All the authors participated in the design, acquisition, analysis and interpretation of data. QC drafted the article and SW and JHL revised it critically for important content. All the authors read and approved the final manuscript.

Funding

None.

Availability of data and materials

The datasets used and/or analyzed during the current study are available from the publicly available SEER database.

Ethics approval and consent to participate

This study was deemed exempt by the Ethics Committee of Peking Union Medical College Hospital, Beijing, China, since the data we used were obtained from the publicly available SEER database.

Consent for publication

No conflict.

Competing interests

The authors declare that they have no competing interest.

Received: 29 June 2020 Accepted: 7 October 2020

Published online: 17 October 2020

References

1. Machida H, Matsuo K, Yamagami W, Ebina Y, Kobayashi Y, Tabata T, et al. Trends and characteristics of epithelial ovarian cancer in Japan between 2002 and 2015: a JSGO-JSOG joint study. Gynecol Oncol. 2019;153:589-96. 
2. Kobel M, Kalloger SE, Huntsman DG, Santos JL, Swenerton KD, Seidman JD, et al. Differences in tumor type in low-stage versus high-stage ovarian carcinomas. Int J Gynecol Pathol. 2010;29:203-11.

3. Hogen L, Vicus D, Ferguson SE, Gien LT, Nofech-Mozes S, Lennox GK, et al. Patterns of recurrence and impact on survival in patients with clear cell ovarian carcinoma. Int J Gynecol Cancer. 2019;29:1164-9.

4. Lee HY, Hong JH, Byun JH, Kim HJ, Baek SK, Kim JY, et al. Clinical characteristics of clear cell ovarian Cancer: a retrospective multicenter experience of 308 patients in South Korea. Cancer Res Treat. 2020;52:277-83.

5. Cohen JG, Prendergast E, Geddings JE, Walts AE, Agadjanian H, Hisada Y, et al. Evaluation of venous thrombosis and tissue factor in epithelial ovarian cancer. Gynecol Oncol. 2017;146:146-52.

6. Zhang L, Yan L, Niu H, Ma J, Yuan BY, Chen YH, et al. A nomogram to predict prognosis of patients with unresected hepatocellular carcinoma undergoing radiotherapy: a population-based study. J Cancer. 2019;10: 4564-73.

7. Pan X, Yang W, Chen Y, Tong L, Li C, Li H. Nomogram for predicting the overall survival of patients with inflammatory breast cancer: a SEER-based study. Breast. 2019;47:56-61.

8. Hu JQ, Yu PC, Shi X, Liu WL, Zhang TT, Lei BW, et al. Prognostic Nomograms for predicting overall survival and Cancer-specific survival of patients with major salivary gland Mucoepidermoid carcinoma. J Cancer. 2019;10:4380-8.

9. Surveillance, Epidemiology, and End Results (SEER) Program:Incidence- SEER 18 Regs Custom Data (with additional treatment fields), Nov 2018 Sub (1975-2016 varying) National Cancer Institute, DCCPS, Surveillance Research Program, released April 2019, based on the November 2018 submission. https://seer.cancer.gov/.

10. Vickers AJ, Elkin EB. Decision curve analysis: a novel method for evaluating prediction models. Med Decis Mak. 2006;26:565-74.

11. Pawelec $G$. Does patient age influence anti-cancer immunity? Semin Immunopathol. 2019;41:125-31.

12. Prat J. Ovarian, fallopian tube and peritoneal cancer staging: rationale and explanation of new FIGO staging 2013. Best Pract Res Clin Obstet Gynaecol. 2015;29:858-69

13. Yamazaki H, Todo Y, Shimada C, Takeshita S, Minobe S, Okamoto K, et al. Therapeutic significance of full lymphadenectomy in early-stage ovarian clear cell carcinoma. J Gynecol Oncol. 2018;29:e19.

14. Mahdi H, Moslemi-Kebria M, Levinson KL, Gojayev A, Lockhart D, Ali-Fehmi $\mathrm{R}$, et al. Prevalence and prognostic impact of lymphadenectomy and lymph node metastasis in clinically early-stage ovarian clear cell carcinoma. Int J Gynecol Cancer. 2013;23:1226-30.

15. Takei Y, Takahashi S, Machida S, Taneichi A, Yoshiba T, Takahashi Y, et al. Impact of the number of removed lymph nodes on recurrence-free survival in stage I ovarian clear cell carcinoma. Int J Clin Oncol. 2018;23:930-5.

16. Magazzino F, Katsaros D, Ottaiano A, Gadducci A, Pisano C, Sorio R, et al. Surgical and medical treatment of clear cell ovarian cancer: results from the multicenter Italian trials in ovarian Cancer (MITO) 9 retrospective study. Int J Gynecol Cancer. 2011;21:1063-70.

17. Kajiyama H, Suzuki S, Yoshikawa N, Tamauchi S, Shibata K, Kikkawa F. The impact of systematic retroperitoneal lymphadenectomy on long-term oncologic outcome of women with advanced ovarian clear-cell carcinoma. J Gynecol Oncol. 2020.

18. Takahashi K, Takenaka M, Kawabata A, Yanaihara N, Okamoto A. Rethinking of treatment strategies and clinical management in ovarian clear cell carcinoma. Int J Clin Oncol. 2020;25:425-31.

19. Pectasides D, Fountzilas G, Aravantinos G, Kalofonos C, Efstathiou H, Farmakis D, et al. Advanced stage clear-cell epithelial ovarian cancer: the Hellenic cooperative oncology group experience. Gynecol Oncol. 2006;102: 285-91.

20. Chang HT, Chiu ML, Wang TY, Chen TC, Chang CL, Su TH, et al. Effect of chemotherapy, laparoscopy, and cytology on stage IC ovarian clear cell carcinoma: a long-term, single-center study. Int J Environ Res Public Health. 2020;17.

21. Nasioudis D, Mastroyannis SA, Albright BB, Haggerty AF, Ko EM, Latif NA. Adjuvant chemotherapy for stage I ovarian clear cell carcinoma: patterns of use and outcomes. Gynecol Oncol. 2018;150:14-8.

22. Bogani G, Ditto A, Lopez S, Bertolina F, Murgia F, Pinelli C, et al. Adjuvant chemotherapy vs. observation in stage I clear cell ovarian carcinoma: a systematic review and meta-analysis. Gynecol Oncol. 2020;157:293-8.

23. Sugiyama T, Okamoto A, Enomoto T, Hamano T, Aotani E, Terao Y, et al. Randomized phase III trial of Irinotecan plus Cisplatin compared with paclitaxel plus carboplatin as first-line chemotherapy for ovarian clear cell carcinoma: JGOG3017/GCIG trial. J Clin Oncol. 2016;34:2881-7.

24. Diao JD, Ma LX, Sun MY, Wu CJ, Wang LJ, Liu YL, et al. Construction and validation of a nomogram to predict overall survival in patients with inflammatory breast cancer. Cancer Med. 2019;8:5600-8.

25. Zhou Z, Mo S, Dai W, Xiang W, Han L, Li Q, et al. Prognostic nomograms for predicting cause-specific survival and overall survival of stage I-III colon cancer patients: a large population-based study. Cancer Cell Int. 2019;19:355.

26. Wo Y, Yang H, Zhang Y, Wo J. Development and external validation of a Nomogram for predicting survival in patients with stage IA non-small cell lung Cancer $</=2 \mathrm{~cm}$ undergoing sublobectomy. Front Oncol. 2019;9:1385.

27. Kuroda T, Kohno T. Precision medicine for ovarian clear cell carcinoma based on gene alterations. Int J Clin Oncol. 2020;25:419-24.

\section{Publisher's Note}

Springer Nature remains neutral with regard to jurisdictional claims in published maps and institutional affiliations.
Ready to submit your research? Choose BMC and benefit from:

- fast, convenient online submission

- thorough peer review by experienced researchers in your field

- rapid publication on acceptance

- support for research data, including large and complex data types

- gold Open Access which fosters wider collaboration and increased citations

- maximum visibility for your research: over $100 \mathrm{M}$ website views per year

At BMC, research is always in progress.

Learn more biomedcentral.com/submissions 